This item was submitted to Loughborough's Research Repository by the author.

Items in Figshare are protected by copyright, with all rights reserved, unless otherwise indicated.

\title{
Clothing comfort during physical exercise - Determining the critical factors
}

PLEASE CITE THE PUBLISHED VERSION

https://doi.org/10.1016/j.apergo.2018.05.014

PUBLISHER

(c) Elsevier

VERSION

AM (Accepted Manuscript)

PUBLISHER STATEMENT

This work is made available according to the conditions of the Creative Commons Attribution-NonCommercialNoDerivatives 4.0 International (CC BY-NC-ND 4.0) licence. Full details of this licence are available at: https://creativecommons.org/licenses/by-nc-nd/4.0/

\section{LICENCE}

CC BY-NC-ND 4.0

\section{REPOSITORY RECORD}

Raccuglia, Margherita, Benjamin Sales, Christian Heyde, George Havenith, and Simon Hodder. 2018. "Clothing Comfort During Physical Exercise - Determining the Critical Factors". Loughborough University. https://hdl.handle.net/2134/33466. 
Clothing comfort during physical exercise - Determining the critical factors Margherita Raccuglia ${ }^{1}$, Benjamin Sales ${ }^{1}$, Christian Heyde ${ }^{2}$, George Havenith ${ }^{1}$ \& Simon Hodder $^{1}$

${ }^{1}$ Environmental Ergonomics Research Centre, Loughborough Design School, Loughborough University, Loughborough, UK

2 adidas FUTURE Sport Science, Herzogenaurach, Germany

\begin{abstract}
Clothing comfort is determined by multiple material and design factors. Wetness at the skinclothing interface mainly impacts wear comfort. The current study investigated the combined effect of fabric contact area, fabric absolute sweat content and fabric moisture saturation percentage on wetness and stickiness sensations, during exercise. Moreover, factors causing wear (dis)comfort during exercise were identified. Higher fabric saturation percentage induced greater stickiness sensation, despite lower fabric contact area and absolute sweat content (typically associated with lower stickiness). Wetness perception did not change between fabrics with different saturation percentages, contact areas and sweat contents. Therefore, fabric saturation percentage mainly affects stickiness sensation of wet fabrics, overruling the impact of fabric contact area and absolute sweat content. No overall model of wear discomfort across all data could be developed, however, models for different time points were produced, with texture and stickiness sensations being the best predictors of wear discomfort at baseline and during exercise, respectively. This suggests that the factors determining clothing (dis)comfort are dynamics and alter importance during exercise activity.
\end{abstract}




\section{Nomenclature}

ANOVA, analysis of variance

Contact-SA, contact surface area

GSL, gross sweat loss

HIGH, high contact surface area

HR, heart rate

LOW, low contact surface area

MEDIUM, medium contact surface area

Pes, polyester

Sample ABS, sample moisture absorption capacity

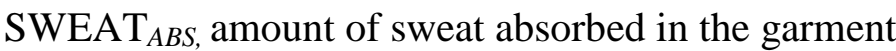

$\mathrm{T}_{\text {core }}$, core temperature 


\section{Introduction}

Comfort is often considered in relation to a single factor causing discomfort, be it environmental, physical, physiological or perceptual (Slater 1986; Kaplan and Okur 2009; Kamalha et al. 2013; Parsons 2014). However, in a real life situation it is rare that only one single factor entirely influences how comfortable an individual feels. Clothing, for instance, constantly interacting with the human body, is responsible for wear discomfort. The clothing system can be considered as a combination of various interacting components that ultimately affect overall clothing functionality and wear comfort sensation. The clothing components can be grouped into two main clusters. The first one is represented by textile factors including the basic yarns and fibres used to knit or weave the fabric, the fabric itself, characterised by different physical parameters (thickness, mass, yarn count, stich density), structures, surfaces and geometries as well as finishes and treatments. The second group includes clothing factors, such as clothing design, fit and openings. Moreover, environmental and individual (including anatomical, physiological, and sensorial) factors interact with the clothing system (Hollies et al. 1979) leading to a highly complex environment- human- clothing system. One of the factors considered as the most crucial in causing wear discomfort during physical activity is the presence of wetness at the skin-clothing interface (Fukazawa and Havenith 2009; Gerrett et al. 2013). The multisensory modality of skin wetness perception contributes to the complexity in studying discomfort during wear. Due to the absence of defined cutaneous sensors (Clark and Edholm 1985) skin wetness is perceived in the central nervous system through the integration of other cutaneous stimulations (Bentley 1900; Niedermann and Rossi 2012; Filingeri et al. 2014). For instance, if the garment that we are wearing becomes wet, a chill or cold feeling will be sensed directly on the skin, due to the cooling effect of evaporation of the liquid and/or the increased thermal conductivity of the fabric. At the same time, the clingy or sticky sensation detected by the cutaneous tactile receptors, 
occurring when the wet material moves intermittently against and across the skin, is combined with the cold sensations in the brain. At this point, the brain, being already familiar with these types of feeling (cold and clingy), recognises the presence of a wet material on the skin (Bentley 1900; Filingeri and Havenith 2015; Bergmann Tiest 2015) resulting in a perception of wetness.

Clothing innovations and advances usually involve the use of textile performance enhancing technologies, validated by standard material test methods conducted with specially developed apparatus. Although these methods allow assessments of objective improvements in material performance, it is often unknown whether these relate to perceivable improvements in wear comfort in real use. The end goal of the clothing industry is to reduce wear discomfort during exercise. Therefore, the adoption of an integrative paradigm where the assessment of textile and clothing parameters (instrumentally measured) is undertaken using human physiological as well as perceptual responses would be of great value. In this regard, recently, in a series of studies in which fabrics were applied to a limited skin area (skin regional studies), the individual and combined roles of fabric thickness (static skin contact) and surface texture (dynamic skin contact) on skin wetness perception was investigated (Raccuglia et al. 2016a; Raccuglia et al. 2016b; Raccuglia et al. 2017a; Raccuglia et al. 2017b). In the static skin application condition, the role of fabric thickness as major determinant of fabric absorption capacity and wetness perception was demonstrated (Raccuglia et al. 2016a; Raccuglia et al. 2016b). Specifically, when applying the same relative to volume water content $\left(\mathrm{mL} \cdot \mathrm{mm}^{-3}\right.$; same saturation percentage) thicker fabrics were perceived wetter than the thinner ones. Conversely, when adding the same absolute water amount $\left(\mathrm{mL} \cdot \mathrm{mm}^{-2}\right)$, thicker fabrics were perceived dryer compared to thinner fabrics, given that thinner fabrics were more saturated. The individuals could perceive various degrees of fabric wetness by integrating fabric thermal (cooling provided) and mechanical (load on the 
skin) inputs sensed at the skin by thermo- and mechanoreceptors, respectively. Specifically, with the increase in fabric water content the cooling power also increases, resulting in higher local skin cooling (reduction in skin temperature) and wetness perception. The contribution of fabric tactile input was indicated by greater wetness perception in heavier fabrics at equal water content, due to the resultant higher load/pressure which increases the magnitude of stimulation of both thermo- and mechanoreceptors. Finally, as expected (Fukazawa and Havenith 2009; Gerrett et al. 2013), sensations of discomfort were strongly correlated to fabric wetness perception, showing the importance of this parameter in overall comfort sensation

In a dynamic skin contact investigation (Raccuglia et al. 2017c; Raccuglia et al. 2017a), i.e. when the fabrics move across the skin, the role of fabric surface properties on wetness perception was studied. It was observed that wet fabric materials with a smoother surface resulted in greater skin wetness perception compared to the wet rougher fabric surfaces. In fact, when moving across the skin, the wet smoother materials may cause higher cutaneous displacement compared to the rougher ones. The higher skin displacement likely resulted from a higher adhesiveness between the wet fabric and skin, which in turn was caused by the creation of a greater number of contact points offered by the smoother fabric surface. The magnitude of skin displacement was detected by the cutaneous tactile receptors as higher or lower stickiness or clinginess sensation and, subsequently associated with different degrees of fabric wetness. Interestingly, the power of wetness perception prediction became substantially stronger when including, both stickiness sensation and fabric thickness as predictors.

Due to the critical impact that stickiness sensation was shown to have on wetness perception in the skin regional study (dynamic contact), the aim of the current study was to investigate the influence of both stickiness sensation and wetness perception on wear 
discomfort, in a whole-body study . In the current study garment wetness was induced by physical exercise (sweating), rather than by manipulating the fabric moisture content by adding water to it, as done in earlier experiments (Raccuglia et al. 2016a; Raccuglia et al. 2017c). The latter difference between whole body (exercise) and the skin regional studies adds an extra different type of thermal sensory cue, which can contribute to the ability to perceive different degrees of fabric wetness. In fact, in the current whole body investigation, the contribution of the cooling effect arising from the evaporation of sweat, induced by physical exercise, was examined. In contrast, in the earlier studies (skin regional study), water evaporation from the fabrics, during the application to the skin, was prevented by covering the fabrics with a thin PVC layer. Therefore, in the skin regional studies, the role of cooling sensations mainly arouse from the increased thermal conductivity of the wetted fabrics.

To solely study the role of fabric stickiness and wetness perception on wear discomfort during physical exercise, three experimental garments were selected and matched for their physical parameters. To induce substantial differences in stickiness and wetness sensations, the fabric surface area in contact with the skin between the three garments, was manipulated. It was hypothesised that higher garment surface area in contact with the skin will result in greater stickiness sensation and wetness perception and that these latter two will impact wear discomfort. However, manipulations of fabric contact surface area could also affect the surface area available for sweat absorption between the three garments, this leading to differences in garments absolute sweat content and moisture saturation. Therefore, the current study examined the combined role fabric contact area with the skin, fabric sweat content and moisture saturation percentage on stickiness sensation and wetness perception. 


\section{Participants}

Eight young (21.4 \pm 2.3 yrs.) males recreationally active (strength and conditioning as well as aerobic exercises at least 4 times per week) and of Western European origin participants, were recruited from the Loughborough University student cohort. Their mean body mass, height and body fat was, $81.0 \pm 10.1 \mathrm{~kg}, 181.1 \pm 8.1 \mathrm{~cm}$ and $15 \pm 3.7 \%$, respectively.

The experimental procedures where fully explained to the participants verbally and in writing, before obtaining informed written consent and completing a health screening questionnaire. All the experimental procedures involved were approved by the Loughborough University Ethical Committee. The study was conducted within the confines of the World Medical Association Declaration of Helsinki for medical research involving human participants.

\section{Method}

\section{Garments}

The experimental garments included three short sleeved 100\% polyester T-shirts with identical design and fit (loose). Three fabrics were selected to produce the three experimental garments. The fabrics were matched for thickness, mass, denier count, fibre content, and finish (hydrophilic finish) (Table 1). The main difference between the three fabrics was in the percentage of surface area in contact with the skin (contact surface area; Contact-SA; Table 1). The latter was achieved by knitting the three fabrics using different mesh structures, each of these characterised by holes of different diameters (Fig 1): high (HIGH), medium (MEDIUM) and low (LOW) Contact-SA. Consequently, these differences in Contact-SA led to differences in surface area available for sweat absorption.

Garment Contact-SA was calculated using a high resolution picture of each fabric with a white background ('holes' area). The creation of high contrast between the fabric and the 
white background allowed measurements of the 'holes area' in unit of pixels, using Adobe Photoshop Software (2017). The Contact-SA was calculated as percentage of the full area of the fabric (in the picture) by subtracting the total area. The Contact-SA expressed as percentage of the 'holes area' was $92.7 \%, 87.5 \%$ and $66.3 \%$, for HIGH, MEDIUM and LOW, respectively.

Due to differences in Contact-SA, the total capacity to absorb liquid moisture (absorption capacity; ABS in $\mathrm{g} \cdot \mathrm{m}^{-2}$ ) was different across the three fabrics (Table1) and therefore garments.

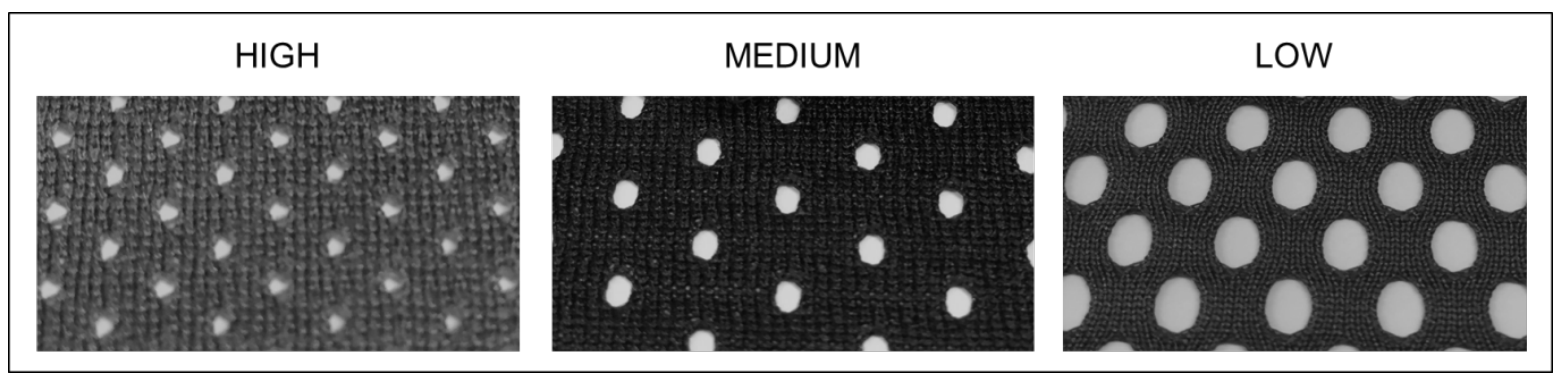

Figure 1 High resolution photographs of HIGH (high Contact-SA), MEDIUM (medium Contact-SA) and LOW (low Contact-SA) fabrics.

Table 1 Experimental fabrics/ garment specifications.

\begin{tabular}{|c|c|c|c|c|c|c|c|}
\hline $\begin{array}{l}\text { Fabrics/ } \\
\text { Garment }\end{array}$ & $\begin{array}{l}\text { Fibre } \\
\text { type }\end{array}$ & $\begin{array}{l}\text { Thickness } \\
\text { (mm) }\end{array}$ & $\begin{array}{c}\text { Fabric } \\
\text { Mass } \\
\left(\mathrm{g} \cdot \mathrm{m}^{-2}\right)\end{array}$ & Structure & $\begin{array}{l}\text { Denier } \\
\text { count }\end{array}$ & $\begin{array}{c}\text { Sample } \\
\text { Contact-SA } \\
(\%)\end{array}$ & $\begin{array}{c}\text { Sample } \\
\text { ABS } \\
\left(\mathrm{g} \cdot \mathrm{m}^{-2}\right)\end{array}$ \\
\hline HIGH & $100 \%$ pes & 0.56 & 85 & $\begin{array}{l}\text { single jersey, } \\
\text { mesh (filament) }\end{array}$ & 50 & 92.7 & 278 \\
\hline MEDIUM & $100 \%$ pes & 0.56 & 85 & $\begin{array}{l}\text { single jersey, } \\
\text { mesh (filament) }\end{array}$ & 50 & 87.5 & 266 \\
\hline LOW & $100 \%$ pes & 0.56 & 80 & $\begin{array}{l}\text { single jersey, } \\
\text { mesh (filament) }\end{array}$ & 50 & 66.3 & 172 \\
\hline
\end{tabular}

HIGH = high contact surface area, MEDIUM = medium contact surface are, LOW = low contact surface area. 100 $\%$ pes $=100 \%$ polyester; Contact-SA $=$ contact surface area calculated with Photoshop Software (2017) subtracting the 'holes area' from the total surface are; $A B S=$ moisture absorption capacity.

\section{Experimental protocol}


Pre-test. Participants were required to attend the Environmental Ergonomics Research Centre for a pre-test, involving anthropometric measurements of height, body mass (Mettler Toledo Kcc150, Mettler Toledo, Leicester, UK), percentage of body fat (Tanita Corporation, Tokyo, Japan) and body dimensions for the anthropometric assessments of the adequate garment size used for the experimental trials.

During the pre-test participants also performed a 20-min running test on a treadmill (h/p/cosmos mercury 4.0, h/p/cosmos Sport \& Medical GmbH, Nussdorf-Traunstein, Germany). During this time the participants were asked to select the speed they could comfortably run for 1 hour. The selected speed $\left(10.2 \pm 0.9 \mathrm{~km} \cdot \mathrm{h}^{-1}\right)$ was then recorded and used for the following experimental trials.

Experimentaltrials. Participants performed 3 running trials on different days, separated by a minimum of 24 hours of rest. In each trial one of the three experimental garments (HIGH, MEDIUM and LOW) was worn. The testing sequence was counterbalanced to minimise any order effect and each participant performed the trials at the same time of the day to minimise circadian variation. Participants ran at the pre-set fixed speed for 30-min. This running duration was selected to replicate the type of activity typically performed in an indoor environment e.g. at the gym. Participants were instructed to refrain from strenuous exercise, abstain from caffeine and alcohol consumption 24 hours before testing, and to keep a record of their food intake and replicate it the day before each visit. In order to maintain euhydration, they were also advised to consume $20 \mathrm{~mL} \cdot \mathrm{kg}^{-1}$ body weight of water during the two hours prior to testing. On arrival to the laboratory participants were asked to void their bladders and self-insert a rectal probe, to monitor changes in body core temperature. The rectal probe (Grant Instrument Ltd, Cambridge, UK) was inserted $10 \mathrm{~cm}$ beyond the anal sphincter and rectal temperature was measured throughout each experimental trial at 1-min intervals and recorded via a portable data logger (Grant Instrument Ltd, Cambridge, UK) 
connected to the thermistor's probe. Participants also wore a wrist-based heart rate (HR) monitor (Polar A360, Polar Electro Oy, Professorintie 5, Kempele, Finland) and HR was recorded before (BASELINE) and during the running trials at 1-min intervals. A wrist-based monitor, rather than a chest-based strap, was used since a chest strap would have interfered with sweat transfer from the skin to the T-shirt. Following from this, semi-nude (including underwear, rectal probe and HR monitor) body mass was recorded. Subsequently, participants were provided with standard running shorts and socks, worn with their personal running shoes, and were asked to use the same personal gears for the entire duration of the experiment. This period of preparation lasted approximately 15-min and allowed time for the stabilisation of HR and $\mathrm{T}_{\text {core }}$. Participants moved to the climatic chamber, rested standing still on the treadmill and after 10-min baseline HR was recorded. They then donned the experimental garment and the running trial started. In order to prevent dehydration, the participants were allowed to drink water ad libitum during the experiment, and liquid consumption was recorded. At the end of the run participants took off the worn T-shirt and hand it over to the experimenter for measurements of post-exercise garment mass. The participants took off shorts, socks and shoes, towelled their skin (this took 2-min) and postexercise semi-nude body mass was recorded.

Sweat production was calculated based on the weight change of each participant (gross sweat loss, GSL), corrected for liquid intake, and reported in grams per body surface area $\left(\mathrm{g} \cdot \mathrm{m}^{-2}\right)$, according to:

$\operatorname{GSL}\left(\mathrm{g} \cdot \mathrm{m}^{-2}\right)=\left[w_{b 1}-\left(w_{b 2}-\right.\right.$ liquid $\left.)\right] / S A$

Where;

$w_{b 1}$ body mass at the start of the experiment (g) 
The experiment was conducted in a climatic chamber maintained at $27.4 \pm 0.3{ }^{\circ} \mathrm{C}, 49.4 \pm 3.4$ $\%$ relative humidity. Apart from having different contact areas, the three garments presented different ventilation potentials, as results of the three different mesh structures. Thus, to cancel-out the effect that the presence of relatively high air flow could have had on sweat evaporation and perceptual responses (i.e. wetness perception, thermal sensation and wear discomfort), environment air flow was set to negligible levels $\left(0.2 \mathrm{~m} \cdot \mathrm{s}^{-1}\right)$.

\section{Perceptual measurements}

During each experimental trial wetness perception, stickiness sensation, thermal sensation, texture sensation and wear discomfort were scored by the participants at 5-min intervals using interval scales, specifically developed by the authors (Raccuglia et al. 2016a; Raccuglia et al. 2017c) (Fig 2). Wetness perception was scored using an ordinal unipolar scale ranging from 0 (extremely dry) to 30 (extremely wet). Stickiness sensation was scored using a 12-points ordinal unipolar scale (0 not -sticky, 12 extremely sticky). Thermal sensation was scored using an ordinal bipolar scale ranging from -10 Cool to 20 Hot, modified from.

In the dynamic skin regional study (Raccuglia et al. 2017c), it was observed an increase in texture sensation when the fabric became wet (i.e. the fabric is perceived rougher than when dry). Interestingly this increase in texture sensation was associated with reductions in pleasantness sensation. Hence, in the current experiment, fabric texture sensations were recorded to assess whether these had an impact also on wear discomfort. Fabric texture sensation was scored with an ordinal bipolar scale (from -9 very smooth to 9 very rough).

Finally, the increase in wear discomfort was scored using an ordinal unipolar scale, ranging from 1 comfortable to 7 very uncomfortable. 


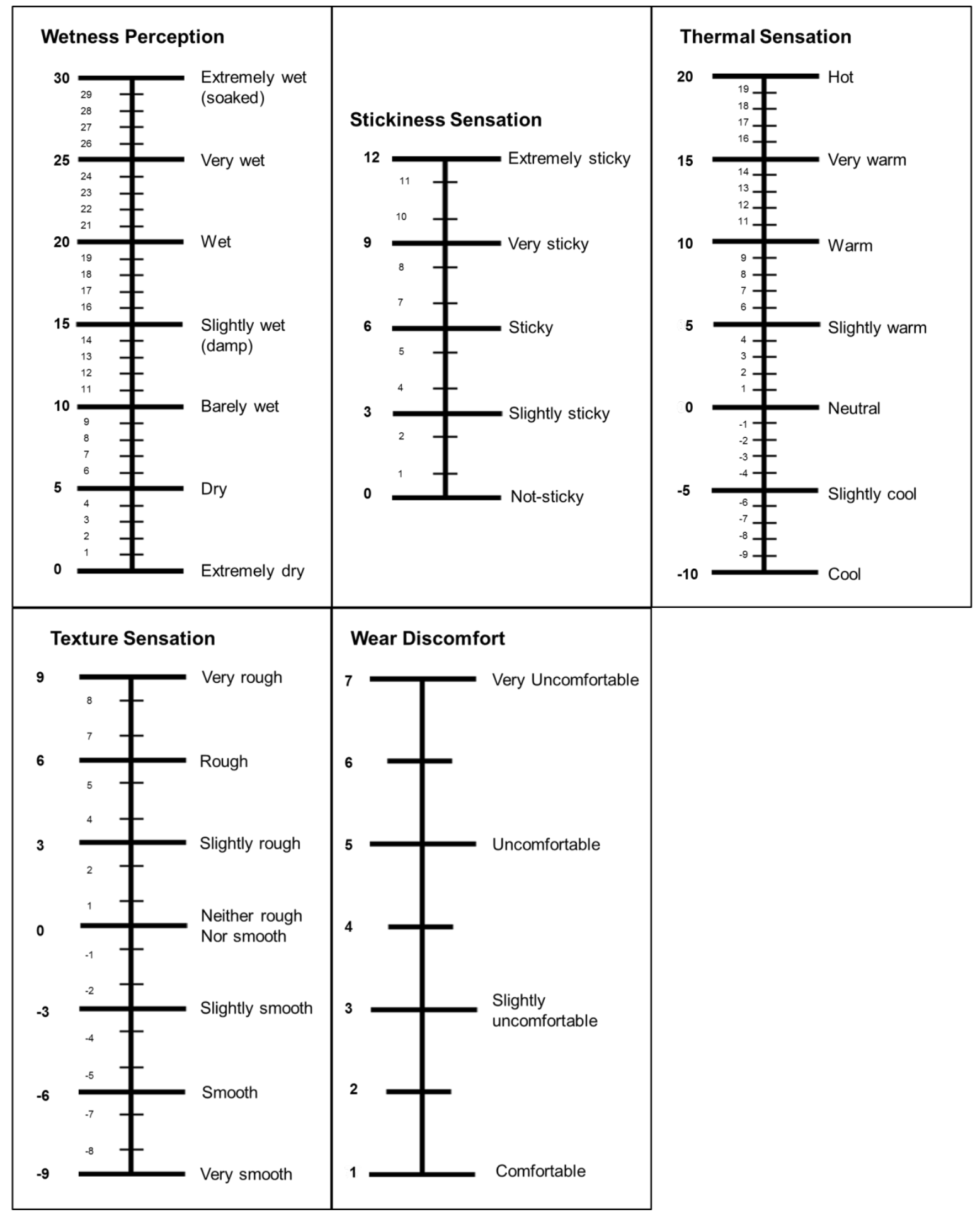

Figure 2 Perceptual scales (Raccuglia et al. 2016a; Raccuglia et al. 2017b). Participants scored each perceptual parameter by reporting verbally the selected number; each score was then recorded by the investigator.

\section{Statistics}

The independent variables were: $\mathrm{HR}$ and $\mathrm{T}_{\text {core }}$ (physiological) and wetness perception, stickiness sensation, thermal sensation, texture sensation and wear discomfort (sensorial). 
Data were tested for normality of distribution and homogeneity of variance with Shapiro-Wilk and Levene’s tests, respectively.

One-way repeated measures ANOVA tests were performed to assess differences in $\mathrm{HR}, \mathrm{T}_{\text {core, }}$ GSL and SWEAT $A B S$ between the garments (HIGH, MEDIUM and LOW). When statistical differences were observed post hoc tests with Bonferroni correction for multiple comparisons were conducted.

Wetness perception, stickiness sensation, texture sensation and wear comfort data were measured through means of ordinal scales and also violated the assumption of normality of distribution, therefore for the statistical analysis non-parametric tests were conducted.

Friedman tests were conducted to assess the effect of garment contact area (HIGH, MEDIUM and LOW) and therefore absolute sweat absorption $\left(\mathrm{g} \cdot \mathrm{m}^{-2}\right)$ as well as saturation (\% of absorption capacity) on wetness perception, stickiness sensation, texture sensation and wear comfort at each of the run time points (BASELINE, 5 MIN, 10 MIN, 15 MIN, 20 MIN, 25 MIN, 30 MIN). When statistical differences were observed Wilcoxon Signed Rank tests were conducted.

To assess the impact of wetness perception, stickiness sensation, thermal sensation and texture sensation (independent variables) on wear discomfort (dependent variable), stepwise regression analyses were performed.

In all analyses $\mathrm{p}<0.05$ was used to establish significant differences. Data are reported as mean \pm standard deviation. Statistical analysis was performed using the software IBM SPSS Statistics version 23 (IBM, Chicago, USA).

\section{Results}

\section{Sweat produced}


The amount of total sweat produced (GSL) at the end of the each run condition was not affected by the type of garment worn, therefore no significant $(p>0.05)$ differences were observed in GSL between HIGH, MEDIUM and LOW (Fig 3).

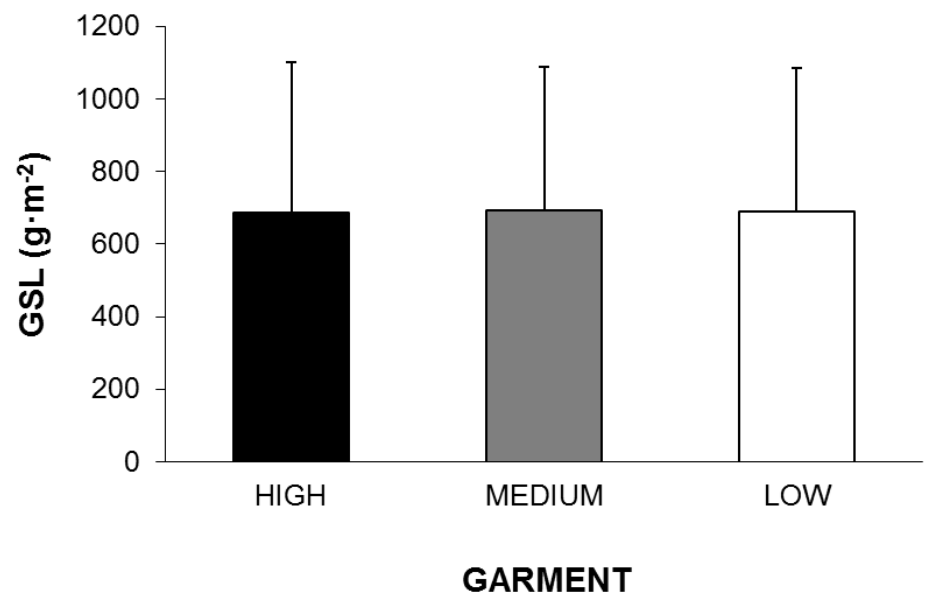

Figure 3 Gross sweat loss (GSL) data between the three experimental garments (HIGH; MEDIUM; LOW). No significant differences in GSL were observed between the three garments.

\section{Heart rate and core temperature}

$\mathrm{HR}$ and $\mathrm{T}_{\text {core }}$ were recorded every 1-min intervals and the average data of 5-min for $\mathrm{HR}$ are reported (Fig 4A). There were no significant differences ( $\mathrm{p}>0.05$ ) in HR (Fig 4A) or $\mathrm{T}_{\text {core }}$ (Fig 4B) between HIGH, MEDIUM and LOW.

A

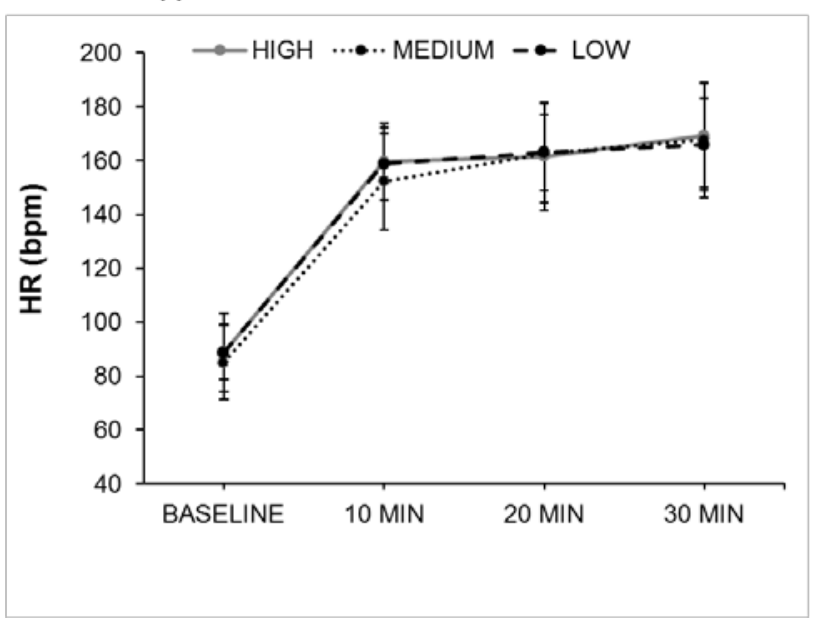

B

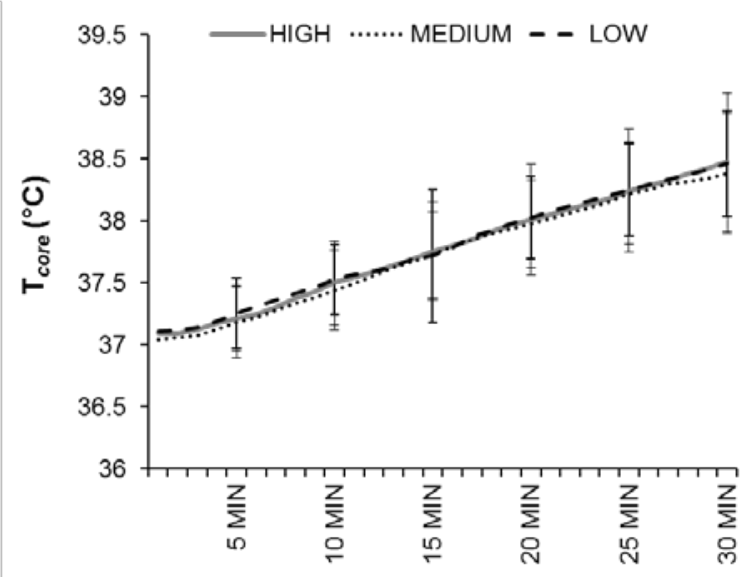

Figure 4 Time course (Baseline - $30 \mathrm{MIN}$ ) of heart rate $(\mathrm{HR} ; 4 \mathrm{~A})$ and core temperature $\left(\mathrm{T}_{\text {core }} ; 4 \mathrm{~B}\right)$. No significant differences In HR or $\mathrm{T}_{\text {core }}$ were observed between the 3 garments (HIGH, MEDIUM and LOW). 


\section{Sweat absorbed}

Garment type had a significant effect $(\mathrm{p}=0.04)$ on sweat absorption (Fig 5A). Specifically, LOW showed a significantly lower $(\mathrm{p}=0.02)$ absolute sweat content $\left(\mathrm{SWEAT}_{A B S}\right)$ compared to HIGH, whereas no significant differences where observed between LOW and MEDIUM (p $=0.09)$, neither between HIGH and MEDIUM $(\mathrm{p}=0.54)$.

LOW was significantly more saturated $(\mathrm{p}=0.01)$ then HIGH (34\% and 21\%, respectively). However, garment saturation in MEDIUM (25\%) was not significantly different from HIGH $(p=0.4)$ or LOW $(p=0.08)($ Fig 5B). Despite the lack of significance, between MEDIUM and LOW a trend was visible for difference in sweat absorption and saturation to develop (p $<0.1)$.
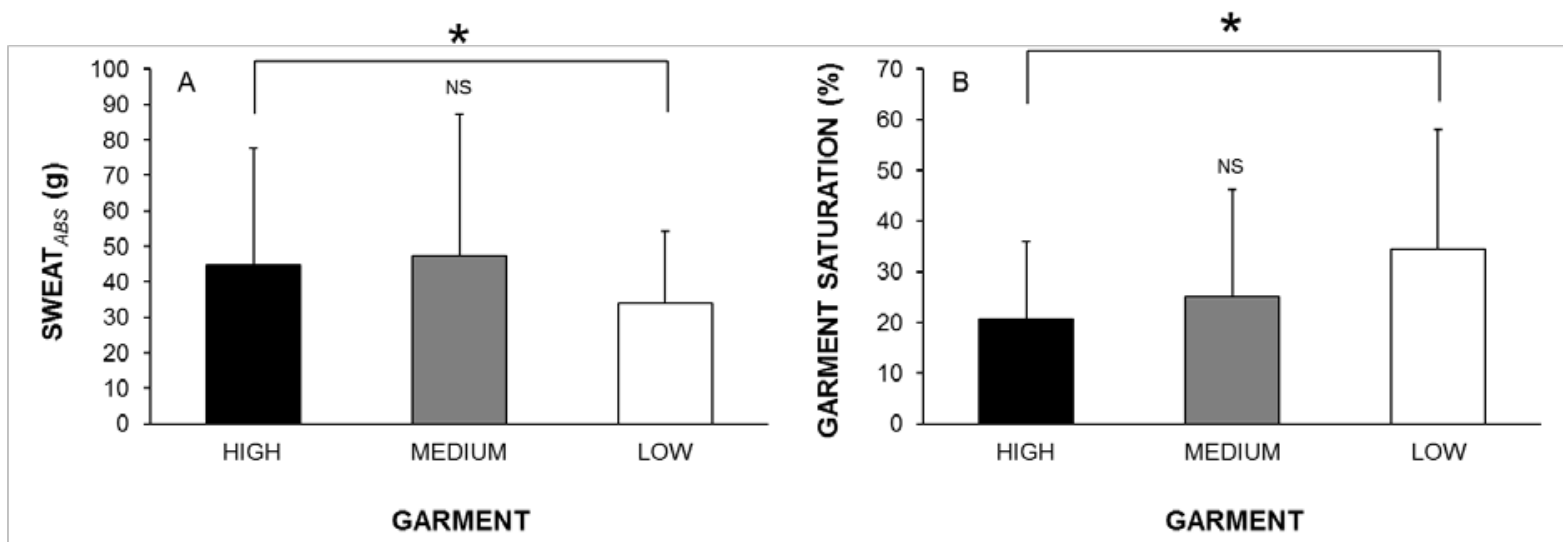

Figure 5A Absolute amount of sweat (SWEAT ${ }_{A B S}$ ) absorbed by the three garments (HIGH, MEDIUM and LOW). Significantly ( ${ }^{*} p=0.02$ ) low SWEAT ${ }_{A B S}$ in LOW compared to HIGH. 5B Percentage of sweat saturation in each garment (HIGH, MEDIUM and LOW). Significantly (* $p=0.01)$ high sweat saturation in LOW compared to $\mathrm{HIGH}$.

Given that garment sweat absorption (SWEAT ${ }_{A B S}$ ) and garment saturation in MEDIUM were not significantly different from HIGH and LOW, MEDIUM was not taken into account for the following analyses.

\section{Wetness perception and stickiness sensation}

Wetness perception (Fig 6A) was not different $(\mathrm{p}>0.05)$ between HIGH and LOW at any of the analysed time points (BASELINE-30 MIN). On the other hand, stickiness sensation (Fig 6B) was significantly different $(\mathrm{p}<0.05)$ between the two garments at each 
time point, apart from BASELINE and 5 MIN ( $>$ > 0.05). Specifically, stickiness sensation was greater in the garment with lower Contact-SA (LOW), which was also more saturated (Fig 5B).

\section{Thermal sensation, texture sensation and wear discomfort}

There were no significant $(\mathrm{p}>0.05$ ) differences in thermal sensation (Fig 6C) between HIGH and LOW. On the other hand, significant differences $(\mathrm{p}<0.05)$ in texture sensation (Fig 6D) were observed between HIGH and LOW at each analysed time point (BASELINE - 30 MIN). Specifically, texture sensation was higher in LOW compared to HIGH (LOW was sensed as more texturized and rougher).

Wear discomfort was significantly higher in LOW compared to HIGH $(\mathrm{p}<0.05)$ at all the analysed time points (Fig 6E). 


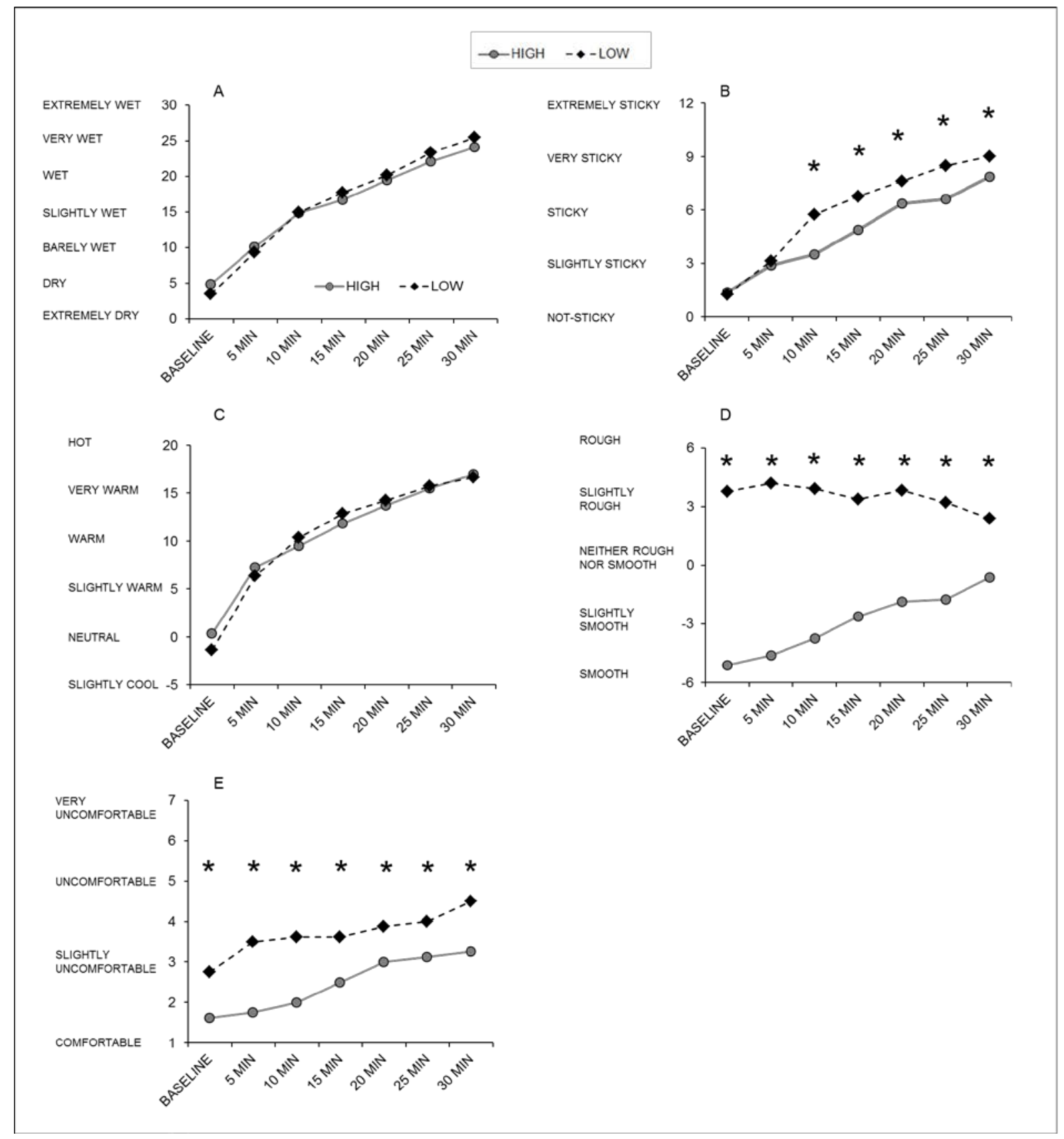

Figure 6 (A) Wetness perception, (B) Stickiness sensation, (C) Thermal sensation, (D) Texture sensation and (E) Wear comfort over time (BASELINE-30 MIN) of the two experimental garments (HIGH and LOW). * indicates significant differences $(p<0.005)$ between the two garments. 


\section{Factors causing discomfort}

Stepwise regression analyses were conducted to identify the factor/s influencing wear discomfort at BASELINE and at 10-, 20- and 30-MIN of exercise activity. For this analysis data from HIGH, MEDIUM and LOW conditions were included. Wetness perception, stickiness sensation, thermal sensation and texture sensation were selected as independent variables and wear discomfort as dependent variable. The analysis was conducted separately for BASELINE, $10 \mathrm{MIN}, 20 \mathrm{MIN}$ and $30 \mathrm{MIN}$ to cancel out a potential time-effect on the prediction models.

Texture sensation was selected as the best predictor of wear discomfort at BASELINE (Fig 7A) $\left(r^{2}=0.30, p=0.019\right)$. Linaer positive relationships were observed between wear discomfort and texture sensation also at 10 MIN (Fig 7B) $\left(r^{2}=0.33, p=0.019\right), 20$ MIN (Fig 7C) $\left(r^{2}=0.28, p=0.019\right)$ and 30 MIN (Fig 7D) $\left(r^{2}=0.22, p=0.018\right)$. However, the power of wear discomfort prediction at 10 MIN (Fig 8B), 20 MIN (Fig 8C) and 30 MIN (Fig 8D), was stronger when selecting stickiness sensation as predictor. Specifically, stickiness sensation explained 36\% $\left(r^{2}=0.36, p=0.02\right), 56 \%\left(r^{2}=0.56, p=0.001\right)$ and $59 \%\left(r^{2}=0.59, p=\right.$ 0.001) of the variance in wear discomfort at $10 \mathrm{MIN}, 20 \mathrm{MIN}$ and $30 \mathrm{MIN}$ of running, respectively. Nevertheless, stickiness sensation did not affected wear discomfort at BASELINE (Fig 8A) $\left(\mathrm{r}^{2}=0.04 \mathrm{p}=0.34\right)$.

A regression analysis for wear discomfort, including all data points did not produce a predictive model. A potential reason for this could be a time effect, i.e. certain parameters are relevant for wear (dis)comfort at specific time points. For this reason, analysis at different experimental stages was performed, and the critical contribution of texture sensation and stickiness at different stages was observed. 

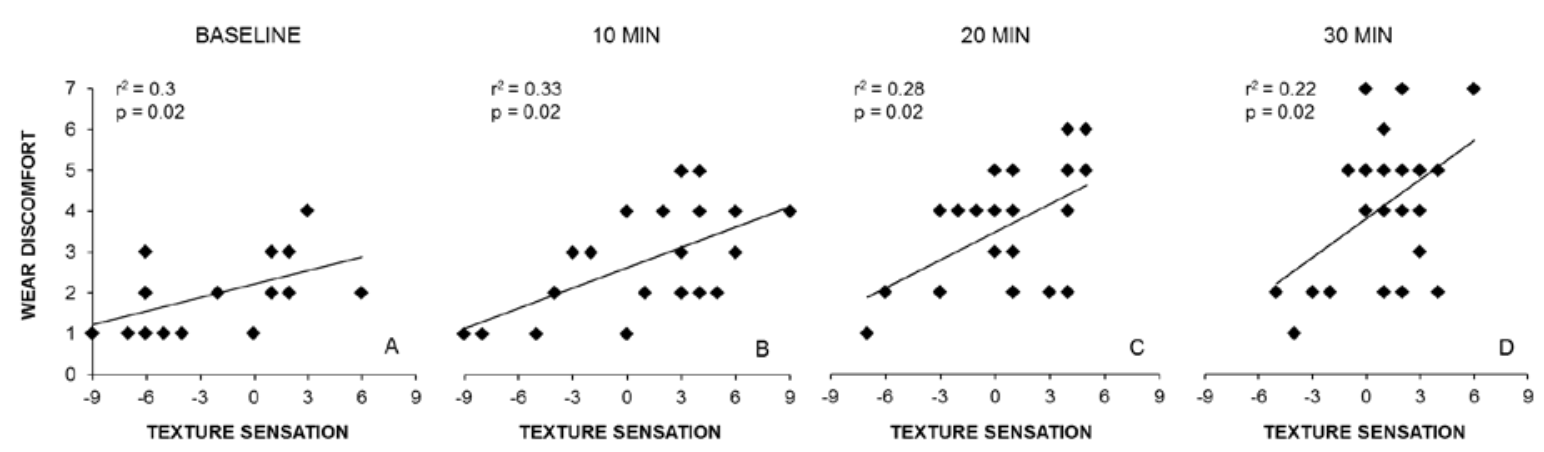

Figure 7 Prediction models of wear discomfort at BASELINE (7A), 10 MIN (7B), 20 MIN (7C) and 30 MIN (7D), using texture sensation as predictor. To predict wear discomfort stepwise linear regression analyses were performed separately for each selected time point.
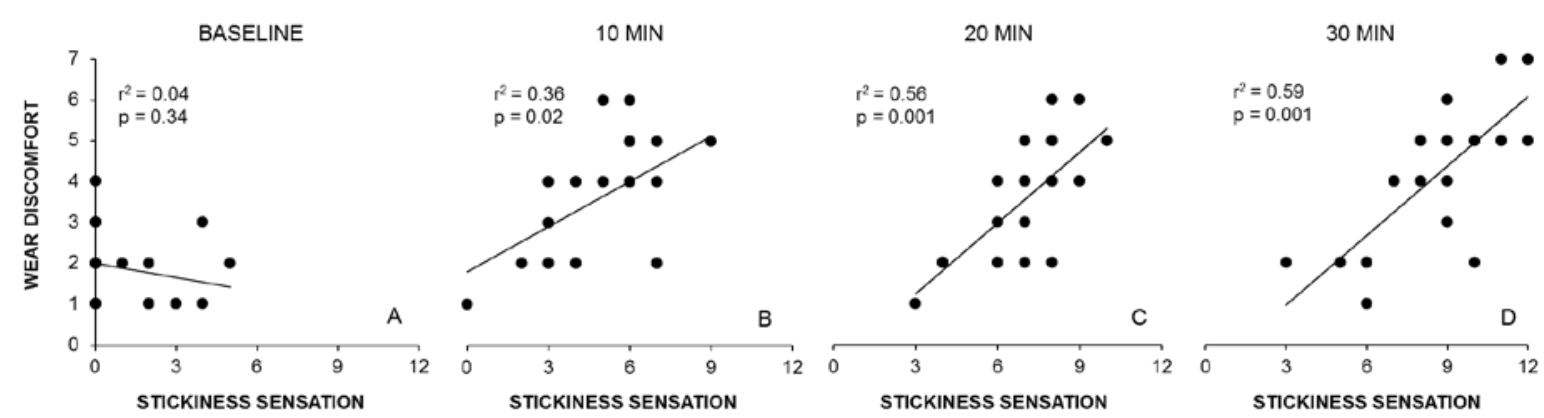

Figure 8 Prediction models of wear discomfort at BASELINE (8A), 10 MIN (8B), 20 MIN (8C) and 30 MIN (8D), using stickiness sensation as predictor. To predict wear discomfort stepwise linear regression analyses were performed separately for each selected time point.

\section{Discussion}

In this study we used an integrative approach where moisture-handling properties of textile materials were assessed through changes in wear comfort during exercise. In particular, the potential impact of garment surface area in contact with the skin on wetness perception and stickiness sensation was investigated. Manipulations of fabric contact area caused differences in the fabric area available for sweat absorption. Consequently, these changes lead to differences in garment sweat content and saturation (percentage of total absorption capacity), the latter affecting perceptual responses. Additionally, the influence of wetness perception, stickiness sensation, thermal sensation and texture sensation on wear discomfort during physical exercise was explored. While in a research-based environment it is common to conduct human wear testing, this experiment clearly demonstrated the value for the clothing 
manufactures to also use a paradigm in which clothing development involves an additional human-focused evaluation process, rather than ending at the material evaluation level.

\section{Sweat produced and sweat absorbed}

The performed running exercises led to the same sweat production between the three experimental conditions (HIGH, MEDIUM and LOW garment) however, different sweat contents were achieved between the garments. Specifically, the LOW contact surface area garment, showed the lowest sweat content (34 g) but, due to the lowest contact surface area available for sweat absorption, it was also the most saturated (34\%). The latter was followed by MEDIUM and HIGH contact surface area garments, which due to the higher contact surface area, presented greater absolute sweat content (47 g and $45 \mathrm{~g}$ respectively) while being less saturated (27\% and $21 \%$, respectively).

As expected, the differences observed in garment sweat content and saturation did not affect the physiological responses of core temperature and heart rate.

\section{Thermal sensation}

Variations in thermal sensation between the garments, which could have occurred as result of different sweat content and ventilation potentials, were successfully controlled. In fact, thermal sensation was not different between the three garments, likely due to the deliberately low air flow in the environment.

\section{The role of fabric contact area with the skin}

In a study where pre-wetted fabrics were applied in dynamic contact with the inner forearm, it was observed that sensations of stickiness of wet fabrics can be exacerbated when using fabrics with smoother surface texture (Raccuglia et al. 2017c). Specifically, when moving across the skin, the wet smoother fabric surfaces would have created higher level of contact with the skin, resulting in higher skin displacement and associated with higher stickiness. According to this, in the current study it was hypothesised that the level of fabric 
surface area in contact with the skin would have had an impact on stickiness sensation and consequently wetness perception, with more contact area increasing stickiness sensation and wetness perception. However, in the current experiment, the opposite was observed, with low fabric contact area inducing higher stickiness sensation. Specifically, when reducing the amount of fabric area in contact with the skin, less fabric area for sweat absorption was available. Indeed, higher moisture saturation percentage was observed in fabric with lower fabric contact area, this subsequently leading to a greater stickiness sensation. Therefore, while fabric surface texture can affect stickiness sensation and related discomfort through changes in fabric contact area with the skin (Raccuglia et al. 2017c), it is also important to consider the consequences of the contact area for moisture absorption and saturation as well. In fact, fabric moisture saturation may be the real cause for the current findings and may have 'overruled' the impact of contact area. Similar to other multifactorial systems (Lloyd et al. 2016), the current findings suggest that in a human-clothing system the integration and also the strength of different cutaneous stimuli, triggered by various textiles parameters, should be considered.

\section{Stickiness sensation and wetness perception}

The differences in saturation and sweat content between the LOW and HIGH garments, achieved through manipulations of fabric contact area (and therefore fabric area available for absorption), resulted in significant differences in stickiness sensation. Specifically, stickiness sensation was higher in the LOW T-Shirt, despite presenting a lower absolute sweat content, compared to the HIGH one. The finding might appear controversial when looking at the absolute data (sweat absorption in grams), however when considering sweat content as percentage of the absorption capacity of the garment (maximum amount of liquid moisture that can be absorbed by the garment), it can be observed that the LOW garment (lower contact surface area and lower absorption capacity) was more saturated than 
HIGH (higher contact surface area and higher absorption capacity), this resulting in greater stickiness sensation. The current findings are in line with recent observations (Raccuglia et al. 2016a), which led to conclude that fabric saturation, rather than the absolute moisture content, is a better parameter to consider when studying moisture properties of fabrics (even if unmatched for thickness or volume).

Despite the significant differences in sweat content, saturation percentage and also stickiness sensation, wetness perception was almost identical between the two garments. The latter was unexpected, since recently a strong positive relationship between stickiness sensation and wetness perception was observed in a skin regional study (Raccuglia et al. 2017c). Wetness and humidity can be perceived on the skin through the combinations of cold sensations (i.e. sweat evaporation and/or contact with a liquid colder than then skin) and tactile sensations (i.e. stickiness or clinginess) (Bergmann Tiest et al. 2012; Filingeri et al. 2014). In the skin regional studies, fabric moisture content was manipulated by adding water at room temperature to the fabric, this substantially increasing the thermal conductivity of the fabric and acting as cold cue for wetness perception. On the other hand, it seems that during exercise in warm ambient temperature, sweat evaporation did not provide enough cooling, typically considered one of the thermal cues involved in the perception of skin wetness (Niedermann and Rossi 2012). Therefore, the current results indicate that when performing physical exercise in warm environments, differences in garment stickiness sensation do not necessarily affect wetness perception. Consequently, in a mild or hot environment, when cold sensory cues are restricted, stickiness sensation seems a more impactful parameter to consider when determining moisture-related differences between garments.

\section{Wear discomfort}

The other aim of the current study was to identify the factor/s influencing discomfort during wear. Whilst no overall model for discomfort across all data could be developed 
(suggesting complex interactions between the relevant parameters and the time-course development of the relationships), models for different time points were produced. The results showed that at baseline, when the individuals just donned the garment, wear discomfort is in part affected by sensations of fabric texture. Specifically, the LOW garment (low contact surface area) was perceived significantly rougher than the HIGH garment (high contact surface area). In fact, the lower contact surface area, given by the presence of larger holes in the LOW fabric, might have led to a fabric with a high number of edges, causing noticeable sensation of roughness. The latter is illustrated in figure $6 \mathrm{D}$, which also suggests that this effect diminishes over time, mostly likely due to the effect of moisture absorbed in different fabrics. The influence of texture sensation on wear discomfort, during the initial interactions of the garment with the skin, explains why garment texture may be a parameter partially affecting the buying decision process of a specific clothing product.

With the start of the physical exercise, another factor appears to contribute to the increase in wear discomfort. In fact at 10-min, 20-min and 30-min of running exercise, stickiness sensation was selected as the main parameter affecting wear discomfort.

Texture sensation explained $30 \%$ of the variance in wear discomfort at baseline whereas during exercise stickiness sensation (at 10-min, 20-min and 30-min) explained approximately $50 \%$ of the variance in wear discomfort. As such, although the current study successfully identified factors that can contribute to wear discomfort at rest and during exercise, a substantial amount of variance in discomfort ( 50\%) still needs to be explained. The achieved predictive power, however, seems reasonable, given the complexity of the environment-human-clothing system in which the sensation of wear comfort results from the interaction of a number of different factors. For this reason, future investigations should address the role that other parameters could play on wear discomfort, to achieve more accurate estimations of wear comfort and to improve clothing performance. 


\section{Conclusions}

The current findings indicated that when the reduction in fabric contact area percentage causes a decrease in the area available for sweat absorption i.e. in a mesh material, a fabric with higher sweat saturation percentage is obtained. Higher sweat saturation percentage will then cause greater stickiness sensations, despite lower contact area (typically associated with lower stickiness). This suggests that, when studying moisture-related responses in humans, it is important to consider the interaction of different textile parameters, as in this case the combined effect of fabric contact area and moisture saturation percentage.

Factors influencing perception of wear discomfort can change over time and in relation to the over-time changes in human thermophysiological responses, such as metabolic rate and sweating. These changes will in turn affect moisture and haptics responses in humans, leading to a highly complex model of wetness perception in relation to textile materials. Specifically, during exercise in warm conditions, the achieved changes in garment sweat content and saturation resulted in significant differences in stickiness sensation. Nevertheless, these differences did not impact wetness perception responses in the warm environmental condition examined. In fact, in a warm environment (no air movement) and during exercise, the cold sensations, arising from sweat evaporation, may not be sufficient to allow discriminations between the two moisture levels in the garments. On the other hand, differences in stickiness between the two garments were clearly sensed. This indicates that stickiness sensation might represent a more impactful parameter to consider. Consequently, stickiness sensation was indicated as parameter in part ( 50\%) affecting wear discomfort during physical exercise, whereas wetness perception itself did not significantly contribute to the variation in wear discomfort in the tested conditions. Additionally, during the first contact with the skin, when the garment is still dry, the sensation of fabric texture was indicated as the main parameter contributing to wear (dis)comfort. 
The clothing industry tends to end the development process at the material tests level, due to cost and/or time-related reasons. In line with a research-focused approach, this study highlighted, in human wear testing, the subjective sensations which are sources of wear discomfort during physical exercise. Therefore, in order to assess the impact of textile innovations on wear (dis)comfort, the process of clothing development should include a human evaluation level, rather than ending at the material testing level. This could lead to paradigm shift in clothing development, resulting in a human-orientated design process.

Future studies should account for additional critical factors contributing to wear discomfort during exercise and take into account other environmental conditions (i.e. outdoor cold conditions), in order to advance predictions of wear discomfort and the development of sportswear as well as protective clothing.

When considering the multifactorial nature of wear comfort, complementary data also examining the factors influencing it at the moment of the purchase (shop setting) and postexercise, are necessary. This will potentially lead to a holistic model of wear comfort in an environment-human-clothing system. 


\section{References}

Bentley IM (1900) The Synthetic Experiment. The American Journal of Psychology 11:405425.

Bergmann Tiest WM (2015) Tactual perception of liquid material properties. Vision research 109:178-84.

Bergmann Tiest WM, Kosters ND, Kappers AML, Daanen HAM (2012) Haptic perception of wetness. Acta psychologica 141:159-63.

Clark R, Edholm O (1985) Man and His Thermal Environment. London: E. Arnold.

Filingeri D, Fournet D, Hodder S, Havenith G (2014) Why wet feels wet? A neurophysiological model of human cutaneous wetness sensitivity. Journal of neurophysiology 112:1457-69.

Filingeri D, Havenith G (2015) Human skin wetness perception: psychophysical and neurophysiological bases. Temperature 2:86-104.

Fukazawa T, Havenith G (2009) Differences in comfort perception in relation to local and whole body skin wettedness. European journal of applied physiology 106:15-24.

Gerrett N, Redortier B, Voelcker T, Havenith G (2013) A comparison of galvanic skin conductance and skin wettedness as indicators of thermal discomfort during moderate and high metabolic rates.

Hollies NRS, Custer AG, Morin CJ, Howard ME (1979) A Human Perception Analysis Approach to Clothing Comfort. Textile Research Journal 49:557-564.

Kamalha E, Zeng Y, Mwasiagi JI, Kyatuheire S (2013) The Comfort Dimension; a Review of Perception in Clothing. Journal of Sensory Studies 28:423-444.

Kaplan S, Okur A (2009) Determination of coolness and dampness sensations created by fabrics by forearm test and fabric measurements. Journal of Sensory Studies 24:479_ 497. 
Lloyd A, Raccuglia M, Hodder S, Havenith G (2016) The interaction between environmental temperature and hypoxia on central and peripheral fatigue during high-intensity dynamic knee extension. Journal of Applied Physiology jap.00876.2015. doi: 10.1152/japplphysiol.00876.2015

Niedermann R, Rossi RM (2012) Objective and subjective evaluation of the human thermal sensation of wet fabrics. Textile Research Journal 82:374-384.

Parsons KC (2014) Human thermal environments : the effects of hot, moderate, and cold environments on human health, comfort, and performance, Third Edit. CRC Press, London, UK

Raccuglia M, Hodder S, Havenith G (2016a) Human wetness perception in relation to textile water absorption parameters under static skin contact. Textile Research Journal 87:2449-2463.

Raccuglia M, Hodder S, Havenith G (2016b) Humans' moisture perception of textiles materials under static contact with the skin. In: The FASEB Journal. p 1284.13-1284.13

Raccuglia M, Hodder S, Havenith G (2017a) Human Wetness Perception: From Skin To Clothing. Medicine \& Science in Sport \& Exercise 49:511.

Raccuglia M, Hodder S, Havenith G (2017b) Human wetness perception in relation to textile water absorption parameters under static skin contact. Textile Research Journal 87:2449-2463. doi: 10.1177/0040517516671127

Raccuglia M, Pistak K, Heyde C, et al (2017c) Human wetness perception of fabrics under dynamic skin contact. Textile Research Journal. doi: 10.1177/0040517517716905

Slater K (1986) Discussion paper the assessment of comfort. Journal of the Textile Institute 77:157-171. 